\title{
e-Phaïstos
}

e-Phaïstos

Revue d'histoire des techniques / Journal of the history

of technology

VI-1 2017| 2018

Varia

\section{Le Rôle des traductions scientifiques dans l'essor technique japonais au XIX ${ }^{\mathrm{e}}$ siècle.}

Le cas du "Recueil de méthodes de fabrication du verre" de Baba Sadayoshi.

The Role of Scientific Translations in Japanese Technical Development in the Nineteenth Century. The Case of the "Compendium of Glass Manufacturing Methods" of Baba Sadayoshi.

\section{Céline Zuretti}

\section{(2) OpenEdition}

Journals

Édition électronique

URL : http://journals.openedition.org/ephaistos/3056

DOI : 10.4000/ephaistos.3056

ISSN : 2552-0741

Éditeur

IHMC - Institut d'histoire moderne et contemporaine (UMR 8066)

Référence électronique

Céline Zuretti, "Le Rôle des traductions scientifiques dans l'essor technique japonais au XIXe siècle. », e-Phaïstos [En ligne], VI-1 2017 | 2018, mis en ligne le 27 juin 2018, consulté le 21 avril 2019. URL http://journals.openedition.org/ephaistos/3056 ; DOI : 10.4000/ephaistos.3056

Ce document a été généré automatiquement le 21 avril 2019

Tous droits réservés 


\section{Le Rôle des traductions scientifiques dans l'essor technique japonais au $\mathrm{XIX}^{\mathrm{e}}$ siècle.}

Le cas du "Recueil de méthodes de fabrication du verre" de Baba Sadayoshi.

The Role of Scientific Translations in Japanese Technical Development in the Nineteenth Century. The Case of the "Compendium of Glass Manufacturing Methods" of Baba Sadayoshi.

\section{Céline Zuretti}

1 Dans la première moitié du XIX ${ }^{\mathrm{e}}$ siècle, au Japon, la verrerie de la période prémoderne a connu un nouvel essor. La verrerie des XVII ${ }^{\mathrm{e}}$ et $\mathrm{XVIII}^{\mathrm{e}}$ siècles, appelée biidoro (de vidro : verre en portugais), était déjà une production influencée par les techniques occidentales mais réalisée avec un verre au fort taux de plomb issu de la tradition chinoise et dans des formes très ancrées dans la culture locale. 


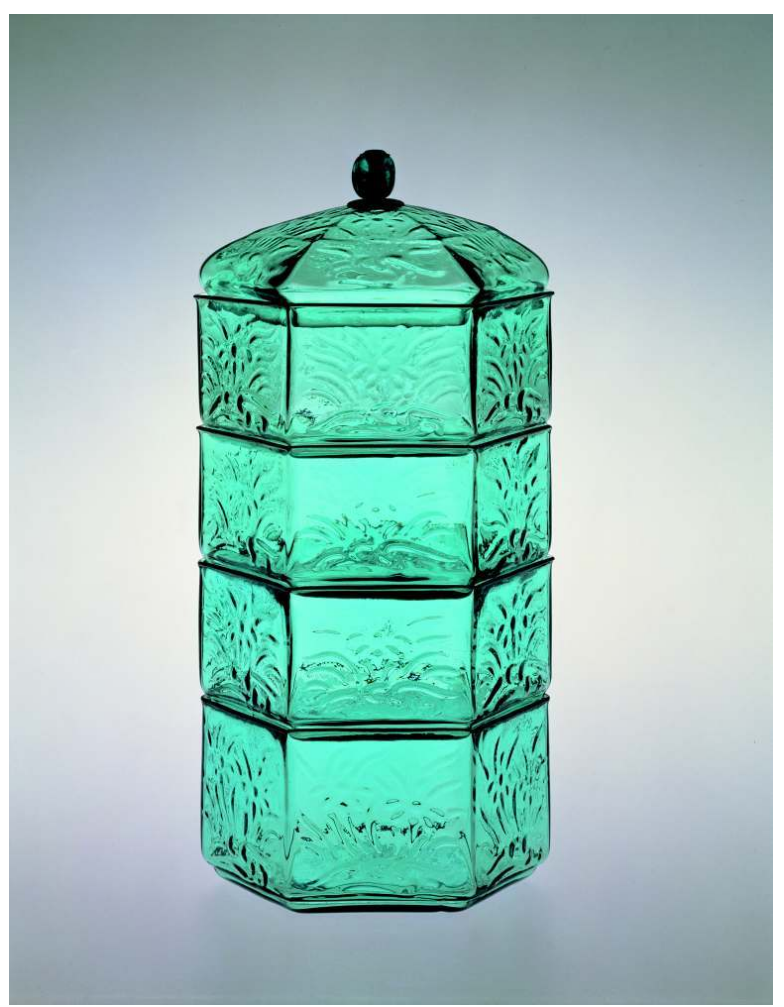

Verre bleu pâle à motif fleuri, soufflé dans un moule, 1714 . Dimensions : $25,5 \mathrm{~cm}$ de haut, $12,7 \mathrm{~cm}$ de diamètre.

Kobe City Museum, publication autorisée pour e-Phaïstos

2 La verrerie de la fin de la période, dite de type giyaman (de diamant en hollandais pour désigner la verrerie taillée), répondait à de nouveaux besoins. Il fallait concurrencer le coût de la verrerie d'art d'importation, fournir de nouveaux contenants pour des produits commerciaux et enfin du matériel de laboratoire, de plus en plus demandé par les savants et médecins pour mener des expériences en chimie notamment, et dont la principale qualité devait être la résistance aux acides. Les sources indiquent que vers 1826, certains verriers parvinrent à créer un verre parfaitement translucide et épais. Vers 1834, ce verre était suffisamment solide pour être taillé à froid et y reproduire des motifs de diamants (incisions décoratives dans la masse du verre) telles que pratiquées dans la verrerie d'art occidentale. Cette production de verre taillé, appelée Kiriko, avait pour fonction de faire directement concurrence aux produits d'importation. Enfin, entre 1845 et 1851, dans le domaine de Satsuma, au sud du Japon, différentes techniques furent maîtrisées comme le soufflage de verre plaqué (superposition d'une couche colorée sur un verre translucide). Ce verre pouvait ensuite être taillé pour faire contraster les différentes couleurs. 


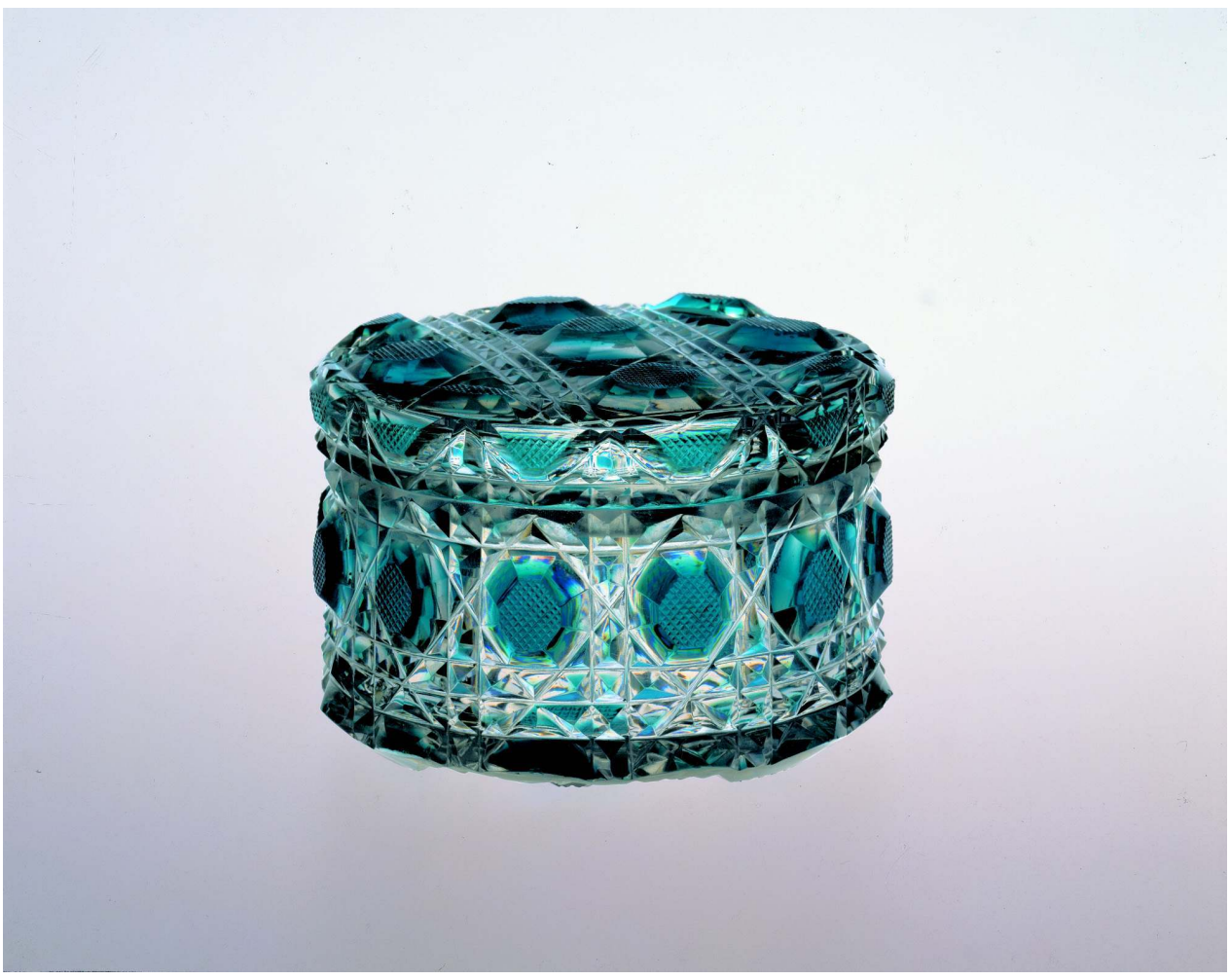

Verre plaqué turquoise sur translucide, taillé avec des motifs en « diamant », 1851-1858. Dimensions $6,8 \mathrm{~cm}$ de haut par $9,8 \mathrm{~cm}$ de diamètre.

Kobe City Museum, publication autorisée pour e-Phaïstos

3 À ce moment, la fabrication d'un verre résistant aux acides fut aussi maitrisée. Cette verrerie se distinguait donc de la première, par la qualité du verre, les techniques de faç onnage de celui-ci et les ressemblances formelles avec les productions occidentales (vaisselle d'agrément, matériel de laboratoire...), ainsi que par la volonté affirmée de concurrencer les produits occidentaux pour accéder à de nouveaux marchés.

Tous ces développements intervinrent alors que le gouvernement shogounal pratiquait encore la politique de fermeture du pays (sakoku). Cette politique fut mise en place vers 1639 et levée en 1854 lors de la signature de traités commerciaux avec différentes nations occidentales. Elle a généralement été comprise comme une sorte de réclusion interdisant tout contact avec l'extérieur. Ce concept a été révisé à partir des années 1970 avec des travaux comme ceux de Ronald Toby dans Re-opening the question of sakoku: Diplomacy in the Legitimation of the Tokugawa Bakufu ${ }^{1}$ et Tashiro Kazui dans Foreign Relations During the Edo Period: Sakoku Reexamined ${ }^{2}$. Ces travaux montrèrent qu'en réalité les échanges diplomatiques et commerciaux avec la Corée, la Chine, le Royaume des Ryūkyū et la Hollande s'étaient maintenus tout au long de cette période. Depuis, de nombreuses recherches sont venues s'ajouter pour relativiser toujours plus cette idée de réclusion.

Une vraie limitation existait cependant au niveau des contacts interpersonnels. La circulation des personnes était strictement surveillée et il est très peu probable que des échanges directs soient survenus entre les Japonais et des artisans verriers occidentaux susceptibles de transmettre les techniques évoquées plus haut. Comment expliquer, dans ce contexte, et en l'absence d'une transmission directe des savoir-faire, les similitudes et 
rapprochements dans les techniques à l'œuvre et les résultats formels, entre les productions verrières du Japon et d'Occident?

On peut trouver un début de réponse en étudiant le travail de traduction effectué, à partir du XVII siècle, par les savants des études hollandaises (rangaku - de ran : abréviation pour Hollande et gaku : étude). Ce groupe de savants (composé d'interprètes de Nagasaki, de m édecins et autres...) s'est intéressé à la culture occidentale, et particulièrement aux sciences (médecine, chimie, artillerie...), jusqu'à former un courant intellectuel à part entière. Ils ont cherché à mettre en pratique diverses techniques et expériences décrites dans les livres qu'ils se procuraient, souvent à grands frais, au port de Nagasaki. En effet, la fermeture n'affectait pas la circulation de nombreux produits commerciaux dont faisaient partie les livres, tant qu'ils n'avaient pas trait, de près ou de loin, au christianisme. À partir de la fin du XVIII ${ }^{e}$ siècle, les savants des études hollandaises ont redoublé d'efforts pour traduire des ouvrages sur les sujets qui les intéressaient, plus particulièrement les ouvrages traitant des sciences et des techniques.

7 En prenant pour objet d'étude la traduction de l'interprète Baba Sadayoshi (1787-1822) intitulée Recueil de méthodes de fabrication du verre ${ }^{3}$ (Biidoro seihō shūsetsu), de 1810, nous souhaitons souligner la richesse des avancées qu'a permis la filtration des livres, ainsi que l'importance déterminante des traductions scientifiques dans l'essor de la verrerie au Japon à la fin de la période prémoderne. Cet angle d'étude soulève les questions suivantes : Quels étaient les livres traduits? Y a-t-il une corrélation entre le contenu des traductions et les méthodes mises en pratique pour la production de cette nouvelle verrerie ? Et si oui, de quelle façon ces méthodes sont-elles parvenues, de la sphère de la littérature scientifique des savants, à celle des savoir-faire artisanaux ?

Depuis les travaux de Dorothy Blair ${ }^{4}$ et de Tanahashi ${ }^{5}$ sur la verrerie, dans les années 1970, le rôle des savants des études hollandaises dans le développement des techniques du début du XIX siècle est connue, mais mal évalué selon nous. Dorothy Blair souligne par exemple l'importance fondamentale de Sakuma Shōzan (1811-1864), un savant des études hollandaise qui expérimenta avec le verre dans les années $1844-45^{6}$. Elle omet, cependant, totalement le travail de ses prédécesseurs : Baba Sadayoshi, dont nous allons étudier le travail ici, et Udagawa Yōan (1798-1866), qui a traduit sous le titre de Premiers principes de Chimie (Seimi Kaiso ${ }^{7}$ ), entre 1837 et 1847, la version hollandaise de Elements of Experimental Chemistry (1799) du chimiste William Henry (1774-1836).

9 Inoue Akiko mentionne très brièvement l'introduction des savoirs occidentaux au moyen des livres en hollandais à la fin du XVIII e siècle et souligne l'importance du Recueil de méthodes de fabrication du verre de Baba Sadayoshi, mais n'apporte aucune précision ${ }^{8}$. Martha Chaiklin, plus récemment, s'est aussi intéressée à la verrerie du point de vue de l'histoire de la culture matérielle, soit la pénétration des objets occidentaux dans la culture matérielle de la société prémoderne japonaise. Elle mentionne rapidement Baba Sadayoshi et Udagawa Yōan, mais met, elle aussi, l'accent sur Sakuma Shōzan sans introduire de nouveaux éléments sur le rôle de ces traductions?.

10 Nous pensons que le rôle de Sakuma Shōzan a retenu l'attention des chercheurs car ses écrits plus accessibles ont marqué les esprits, éclipsant quelque peu le rôle par ses préd écesseurs. C'est pourquoi nous avons souhaité réexaminer un ouvrage comme le Recueil de méthodes de fabrication du verre écrit trois décennies avant que Sakuma Shōzan ne se lance dans le projet de fabriquer un verre semblable à celui des Occidentaux. 
11 Nous allons donc dans un premier temps rendre compte de l'étude que nous avons réalis ée sur l'ouvrage de Baba Sadayoshi. Après une brève présentation de l'auteur, nous verrons sur quels ouvrages il s'est basé pour réaliser ce manuscrit. Dans un second temps, nous vérifierons s'il existe une corrélation entre ses traductions et les techniques qui ont permis de créer la verrerie du XIXe siècle dans les décennies suivantes. Enfin nous tenterons de mesurer la diffusion que son travail a connue et l'impact réel qu'il a pu avoir sur le savoir-faire des artisans verriers.

\section{Le choix de traduction de Baba Sadayoshi}

Baba Sadayoshi (1787-1822) était un linguiste exceptionnel. Il fut adopté à l'âge de 16 ans par une famille d'interprètes de Nagasaki et formé pour participer au négoce familial. Il a appris le hollandais et l'anglais auprès de l'un des chefs du comptoir hollandais situé sur l'île artificielle de Dejima, dans le port de Nagasaki. Du fait de son excellence, il fit partie de la première équipe de traducteurs officiellement reconnue par le shogunat et installée à partir de 1808 à l'observatoire astronomique d'Edo (aujourd'hui Tokyo). Le Bureau de traduction des livres occidentaux (Bansho wage goyō) regroupait des savants des études hollandaises provenant principalement du milieu des interprètes de Nagasaki et de celui des médecins. Baba Sadayoshi y travailla une quinzaine d'années, assurant, en sus de ses traductions, quelques missions diplomatiques requérant sa connaissance de l'anglais et du russe. Bien que décédé à l'âge de trente cinq ans, il a eu le temps de traduire une cinquantaine d'ouvrages, touchant des sujets très variés (agriculture, vaccination, chimie, industrie, médecine, linguistique...).

Les deux ouvrages sur la chimie traitent du verre. Peut-être faut-il y voir l'intérêt que suscitait alors ce matériau chez les savants. Nous présentons ici le Recueil de méthodes de fabrication du verre (Biidoro seihō shūsetsu) qu'il a traduit en 1810. Il existe aujourd'hui trois exemplaires manuscrits de cet ouvrage en trois tomes. Nous avons utilisé les exemplaires de l'Université de Waseda ainsi que la transcription de l'exemplaire conservé au Seikadō bunko intégralement publiée par Tanahashi Junji ${ }^{10}$.

14 Les préfaces varient d'un exemplaire à l'autre, mais dans l'une d'elles, Baba Sadayoshi explique avoir « reçu l'ordre [du Shogun] de rechercher dans les ouvrages de ce pays [Hollande] des méthodes de fabrication du verre en Occident et d'en présenter une traduction. [...] j'ai recherché et extrait absolument tout ce qui avait trait aux méthodes de fabrication du verre publiées dans les livres hollandais. » Il précise avoir « [...] obtenu toutes ces méthodes [...] dans les livres des trois savants suivant : Boisu [Buys], Shomeru [Chomel] et Kerekuru [Le Clercq ${ }^{11}$.»

Le contenu est divisé en trois parties correspondant aux trois ouvrages occidentaux desquels Baba Sadayoshi a extrait les passages relatifs au verre (composition chimique du matériau) et à la verrerie (mise en œuvre et organisation de l'atelier ou de la manufacture). Le manuscrit du Seikadō bunko contient, en sus, des reproductions au pinceau des gravures illustrant deux des ouvrages originaux 
Les Souffleurs de vers dans l'atelier (Der Blaazer aan der Arbeid)

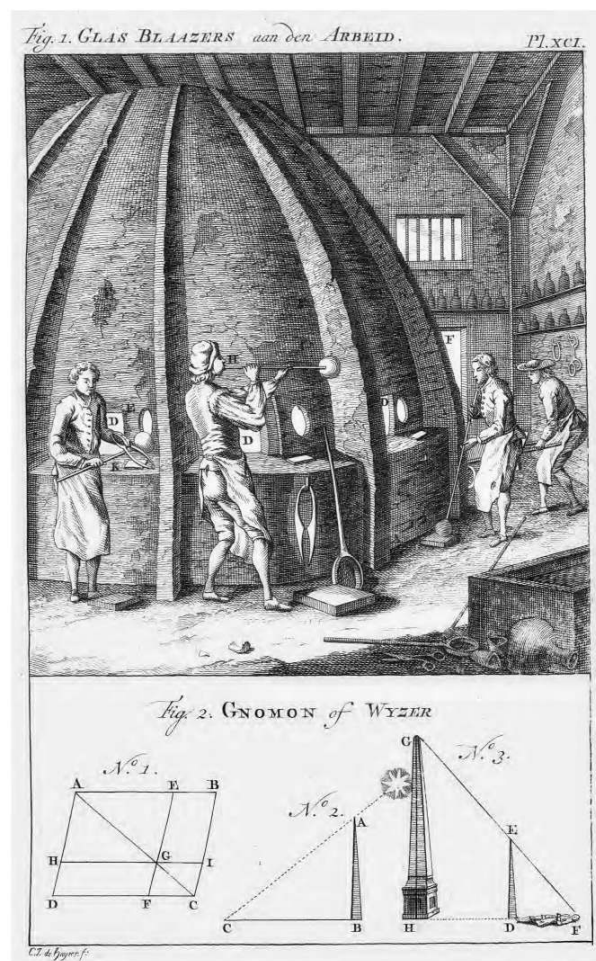

Gravure illustrant Le Nouveau dictionnaire complet des arts et des sciences - tome 4/10 par BUYS Egbert, Amsterdam, 1769.

Koninklijke Bibliotheek, collection numérique, libre de droit 


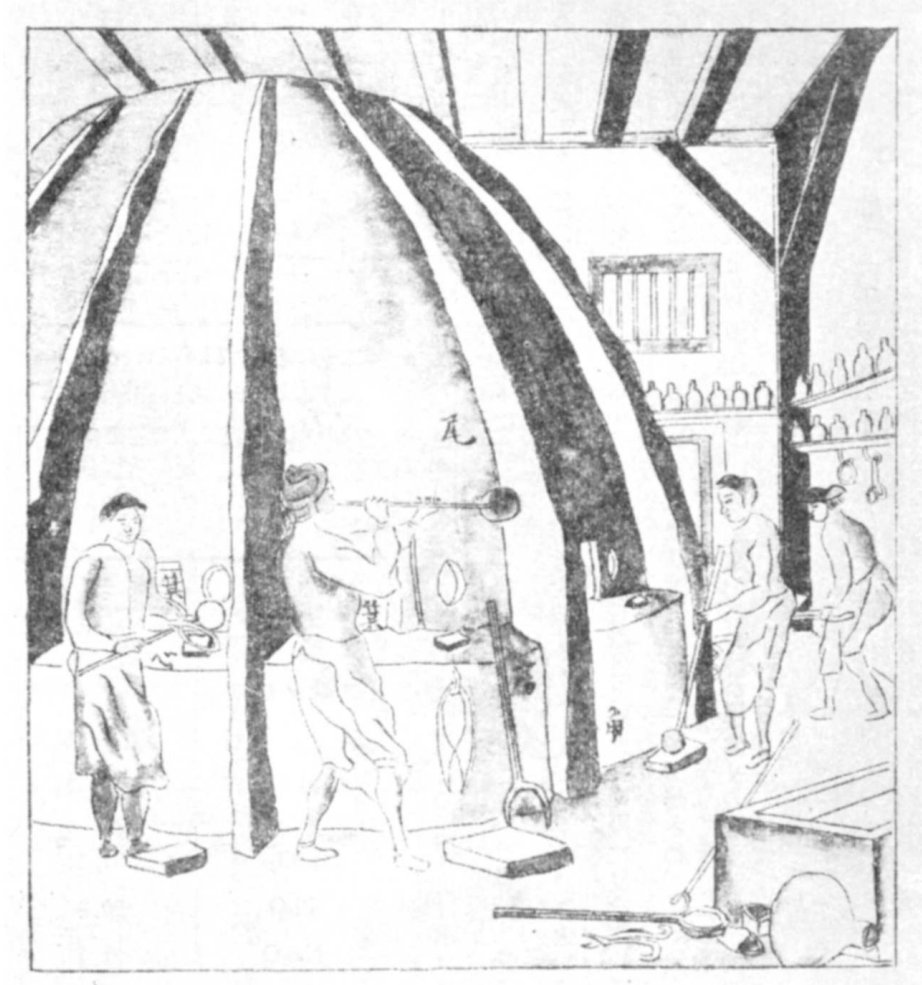

Relevé du dessin illustrant le Recueil de fabrication du verre (Biidoro seihō shūsetsu) de BABA Sadayoshi, 1810, conservé au Seikadō bunko, Japon.

Céline Zuretti

Le Dictionnaire Economique de Noël Chomel, est d'abord paru en français, puis a été traduit en hollandais et augmenté par Jacques Alexandre de Chalmot (1730-1801 $\left.{ }^{12}\right)$. La première parution du dictionnaire remonte à 1709 en France. Ce livre fut traduit dans différentes langues et réédité à plusieurs reprises jusqu'en 1786. On peut parler de succès éditorial européen, voire mondial, puisque sa renommée est même parvenue jusqu'au Japon où c'était un ouvrage très en vogue dans les milieux savants, de la fin du XVIII ${ }^{\mathrm{e}}$ siècle jusque bien avancé dans le XIX ${ }^{e}$ siècle. Il était connu comme "L'Encyclopédie de Chomel ». C'est d'ailleurs la première traduction d'envergure qui fut demandée, en 1811, à l'équipe de traducteurs du gouvernement shogounal. La traduction ne fut achevée que très tardivement, ce qui explique que même en 1844, Sakuma Shōzan ait dû faire importer un exemplaire pour profiter de son contenu. Baba Sadayoshi a tiré de l'Encyclopédie de Chomel 30 articles et recettes traitant du verre. Ces articles abordent la préparation des ingrédients du verre (sable, silex, sels...), la description des gestes techniques pour la réalisation d'objets soufflés et de verre à plat avec la technique des cives (utilisée depuis le Moyen-Âge). Ils comprennent aussi un nombre important de recettes relatives à l'obtention de certaines couleurs par l'ajout d'oxydes métalliques. 
18 Le Nouveau dictionnaire complet des arts et des sciences - tome $4 / 10^{13}$ de Egbert Buys (1725-1769), parut en 1772, est une adaptation basée sur le dictionnaire de Willem Séwel (1654-1720). Les articles traitant du verre sont adaptés d'ouvrages antérieurs comme $L$ ' Arte vetraria d'Antonio Neri (1576-1614), de 1612, et The art of Glass par Christopher Merret (1614-1695), de 1662. Les illustrations représentant les différents postes de travail et les fours reprennent d'assez près les illustrations d'Agricola (1492-1555), dans De re metallica (1556) par exemple. Baba Sadayoshi y a choisi la dizaine d'articles décrivant les ingrédients nécessaires à la fabrication du verre, les outils, y compris les fours, les gestes pour le soufflage ou la réalisation de glaces (plaques de verre) en manchon.

19 Enfin, c'est en réalisant cette étude de l'œuvre de Baba Sadayoshi que nous avons récemment pu identifier le troisième ouvrage qu'il avait utilisé. En croisant divers articles et notices bibliographiques, nous avons compris et vérifié qu'il s'agissait de la traduction hollandaise par Pieter Le Clercq (1692-1759) du Spectacle de la nature - tome 7 de l'Abbé Antoine Pluche ${ }^{14}$ (1688-1761). L'ouvrage, d'abord paru en français, entre 1732 et 1742, était destiné à l'éducation des jeunes. Ce fut un succès éditorial européen, notamment du fait de ses nombreuses planches d'illustrations réalisées avec grand soin et mises en relation avec le texte qu'elles illustraient pour une meilleure compréhension des sujets abordés. Il ne s'agissait donc pas d'un ouvrage scientifique à proprement parler, mais d'un vaste ensemble de descriptions sur des sujets très divers (société, métiers, religions, arts, sciences naturelles...). Les neuf passages traduits par Baba Sadayoshi décrivent l'organisation et le fonctionnement de la manufacture des Glaces de Saint Gobain en France au début du XVIII ${ }^{\mathrm{e}}$ siècle.

20 On constate que les ouvrages choisis par Baba Sadayoshi forment un ensemble disparate en terme d'années de parution, donc d'actualité de l'information contenue, mais aussi en terme d'intention de publication. En effet, ces livres n'étaient pas conçus dans le but de permettre véritablement une mise en pratique mais pour faciliter l'acquisition d'une connaissance de type encyclopédique, dans le cas des dictionnaires de Buys et de Chomel, et d'éduquer les jeunes dans le cas du Spectacle de la Nature de l'Abbé Pluche. Nous verrons maintenant s'il existe une corrélation entre cette compilation de textes variés et les procédés mis en œuvre dans la fabrication de la verrerie du XIX ${ }^{\mathrm{e}}$ siècle?

\section{Les techniques tirées du manuscrit}

21 À la lecture du Recueil de méthodes de fabrication du verre, on constate l'introduction d'un certain nombre de nouvelles informations qui en cas d'application assurent un résultat différent de celui de la verrerie biidoro. Ces informations touchent trois aspects: la composition chimique du verre, les gestes techniques et l'environnement de travail.

Le recueil apporte des recettes permettant de produire des verres de différentes qualités. Donc, par cette traduction Baba Sadayoshi rend accessible tout un éventail de nouveaux mélanges d'ingrédients, mais il confronte immanquablement ses lecteurs à la nécessité d'acquérir des connaissances pratiques dans le domaine de la chimie. Les recettes sont plus précises que dans les ouvrages sur le verre écrit jusqu'alors au Japon. On passe d'une réthorique de recettes aux repères empiriques à une formulation scientifique d'expériences du domaine de la chimie. Les matériaux sont évoqués selon une nomenclature propre à cette science. Les mesures sont indiquées avec précision selon les principes de la chimie moderne occidentale, notamment dans l'ouvrage de Buys. 
L'utilisation du borax plutôt que de la silice dans la composition du verre est décrite à trois reprises et mise en relation avec la production d'un verre dur (résistant aux acides) qui est l'un des enjeux de la verrerie du XIX ${ }^{\mathrm{e}}$ siècle. De plus, la palette de recettes de verres colorés tirée des trois livres est très étendue. Enfin, l'importance de travailler avec des ingrédients purs pour ne pas corrompre le résultat est soulignée à plusieurs reprises. Cette pureté des ingrédients permet notamment l'obtention d'un verre parfaitement translucide.

Les gestes décrits dans le texte, ou représentés dans les illustrations reproduites par Baba Sadayoshi, impliquent la coopération de plusieurs artisans travaillant debout, chacun réalisant des gestes spécifiques et complémentaires (Illustration 3 et 4). Deux techniques de refroidissement lent et de recuisson y sont décrites. Celles-ci sont indispensables au travail du verre épais et aux retouches à froid car elles garantissent la solidité des pièces malgré la rudesse du processus de taille. Le refroidissement lent et la recuisson des pièces sont des conditions sine qua non à la production de la verrerie taillé de kiriko. Ainsi les méthodes de travail décrites diffèrent des gestes employés par les artisans de biidoro et sont susceptibles d'enrichir leur pratique pour atteindre les objectifs commerciaux et techniques du marché de la verrerie au XIX ${ }^{e}$ siècle.

Enfin, l'échelle à laquelle se réalise le travail de production du verre est celle de la manufacture. Le nombre de personnes engagées aux différents stades de la production et la dimension des installations (halles, fours, chambres de refroidissement...) n'ont pas de commune mesure avec les ateliers de verrerie japonais d'alors (Illustrations 4 et 5).

\section{Le Verre (Biidoro)}

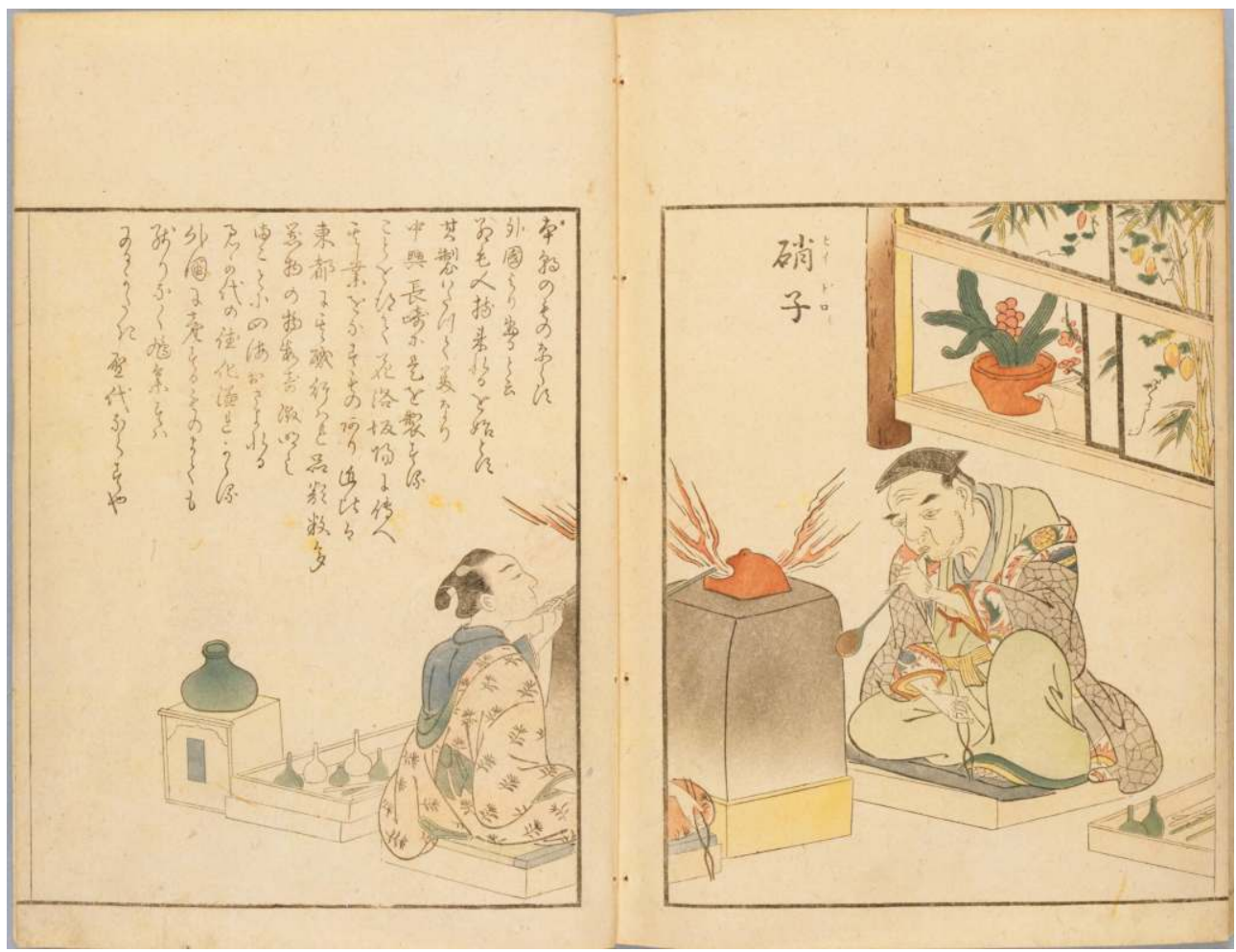

Gravure en couleur illustrant la Classification des artisans (Saiga shikunin burui) par TACHIBANA Minkō, 1770

Bibliothèque de la Diète du Japon, collection numérique, libre de droit 
probablement ce qui a retenu l'attention de Baba Sadayoshi dans la description de la Manufacture des glaces de Saint Gobain dans le Spectacle de la Nature, car par ailleurs, l'auteur de ce livre évite volontairement la description trop détaillée des techniques employées "parce qu'il est juste de conserver aux entrepreneurs les connoissances (sic) auxquelles ils ont un droit exclusif ${ }^{15}$ ». Il a le souci de préserver les détails et secrets qui font le monopole de ce fleuron de la production manufacturière française. La portée pratique de cette description n'est peut-être pas aussi claire que les précédente, mais l'idée de travailler à grande échelle faisait malgré tout son chemin. À partir des années 1840, plusieurs fiefs se sont équipés d'installations de taille plus importante et construites selon des modèles occidentaux comme on le constate dans le fief de Satsuma, au sud du Japon. Ce fief disposait de différents fours de grande taille pour la production de verrerie d'art et de plaques coulées comme décrite dans la traduction de Baba Sadayoshi.

Ainsi, Baba Sadayoshi explique dans sa préface qu'il avait choisi des extraits lui permettant d'exposer en détail des méthodes dont il pensait qu'elles seraient précieuses aux artisans. Cette compilation rassemble donc une somme conséquente d'informations inédites sur la verrerie occidentale. On observe la mise en pratique ultérieure d'une partie de ces informations techniques par des artisans. C'est le cas de l'artisan verrier Kagaya Kyūbei qui annonce sur ses papiers d'emballage publicitaires, en 1826, qu'il fabrique du giyaman (verre épais et translucide). On comprend aussi dans la correspondance de Sakuma Shōzan qu'il a essayé de fabriquer du verre au borax entre 1844 et 1845. Enfin, Kagaya Kamejirō, ancien apprenti de Kagaya Kyūbei a développé, entre 1846 et 1851, dans l'enceinte du projet proto-industriel du Shūseikan, dans le fief de Satsuma, le fameux verre taillé de Satsuma, Satsuma kiriko, le verre plaqué rouge ou bleu sur verre translucide, les plaques de verre moulées développées pour la construction navale et enfin le verre résistant aux acides pour la fabrication des instruments de laboratoire. Il faut souligner que le projet du Shūseikan regroupait diverses activités de production de type manufacturières. La plus part des techniques permettant ce résultat se trouvaient accessibles en langue japonaise dans le Recueil de méthodes de fabrication du verre compilé en 1810 . Nous verrons maintenant quelle a pu être la réception de ce travail et la façon dont il a pu être utilisé.

\section{Diffusion du manuscrit de Baba Sadayoshi}

Il est difficile aujourd'hui d'évaluer avec précision la diffusion qu'a connu le manuscrit et ses différentes copies. Nous disposons, cependant, de quelques indices qui nous permettent dans certains cas d'acquérir une quasi certitude et dans d'autres d'émettre des hypothèses qui demandent encore à être vérifiées.

Nous commencerons par présenter un cas clair de l'utilisation du recueil compilé par Baba Sadayoshi avec l'exemple que nous apporte Sakuma Shōzan dans sa correspondance. Lorsque ce dernier a abordé la lecture de l'Encyclopédie de Chomel, il s'est naturellement confronté à des difficultés linguistiques. Son niveau de hollandais à cette période n'était pas encore très élevé car il avait tout juste commencé à étudier cette langue. Comme Azuma Tōru l'a montré dans Sakuma Shōzan et la Chimie (Sakuma Shōzan to kagaku gijutsu' ${ }^{16}$ ), ses lettres, tant par les choix lexicaux, que par certaines expressions, permettent de comprendre qu'il s'est appuyé sur d'autres ouvrages en japonais pour accéder au contenu de l'Encyclopédie de Chomel. Ainsi les mots quartz, borax, puis le mot silex associés

e-Phaïstos, VI-1 2017| 2018 
systématiquement à l'idée d'un verre dur reflètent ses lectures parallèles. Tantôt il reproduit des imprécisions de traduction de Baba Sadayoshi, tantôt il intercale des explications ou des terminologies provenant de Premier principes de Chimie ${ }^{17}$ par Udagawa Yōan. On comprend donc que trente cinq ans après, le manuscrit est parvenu entre les mains de Sakuma Shōzan, sans doute par le biais de ses relations dans le cercle des études hollandaises.

Or, Sakuma Shōzan a eu recours au savoir-faire d'un artisan verrier pour concrétiser ses expériences. Il décrit comment l'artisan a du prendre le temps de s'adapter à la qualité différente du verre qui perturbait sa maîtrise des gestes techniques habituellement utilisée pour le façonnage du verre au plomb. Cette association d'un artisan et d'un savant, qu'il a décrit dans ses lettres, a certainement été l'occasion d'un échange au cours duquel l'artisan a pu bénéficier des techniques décrites dans un texte auquel il n'aurait sinon pas forcément eu accès. Sakuma Shōzan décrit aussi une autre situation comparable à la sienne. Il écrit : «Récemment, un propriétaire d'atelier de biidoro, qui s'intéresse beaucoup à ces questions [d'un verre semblable à celui des Occidentaux] a tenté à plusieurs reprises d'en fabriquer [du verre dur] avec l'aide d'un médecin spécialiste des études hollandaises mais cela sans succès $^{18}$ \%. Il laisse ainsi entrevoir un artisan en quête de solutions lui permettant d'atteindre les standards occidentaux. C'est donc qu'il y avait un public pour un manuscrit comme le Recueil de méthodes de fabrication du verre et ces rencontres entre les artisans et les savants ont certainement été l'une des voies qui ont permis la diffusion des connaissances de la sphère intellectuelle vers le domaine des savoir-faire artisanaux.

D'autres modes de diffusions se laissent deviner. Par exemple, le manuscrit conservé au Seikadō bunko porte le sceaux de Ōtsuki Gentaku (1757-1827). Ce médecin versé dans les études hollandaises fut nommé au Bureau de traduction des livres occidentaux en 1808, au même moment que Baba Sadayoshi. Ils travaillèrent de concert à la traduction de $\mathrm{l}^{\prime}$ Encyclopédie de Chomel. Ōtsuki Gentaku dirigeait par ailleurs une école privée de hollandais. De tels endroits étaient des lieux où les échanges étaient facilités. Les étudiants pouvaient y consulter la bibliothèque réunie par le maître, apprendre le hollandais, avec des lectures commentées, mais aussi s'essayer à quelque expérience décrite dans les ouvrages occidentaux. Ōtsuki compta parmi ses nombreux disciples un certain Hanai Ikkō (vers 1795 ?) qui travailla le verre et écrivit plusieurs livres sur ce sujet. Dans Le Précis de fabrication du verre (Ruri shōkō zensho), vers 1829, on retrouve certaines des techniques décrites par Baba comme par exemple le refroidissement progressif dans de la poudre de charbon. Ainsi, si le recueil de Baba Sadayoshi n'a pas servit directement aux artisans, l'information qu'il contenait a peut-être été relayée dans une publication plus tardive mais d'une diffusion plus importante.

\section{Conclusion}

31 L'étude du Recueil de méthodes fabrication du verre nous a appris que les extraits choisis par Baba Sadayoshi ne provenaient pas d'ouvrages ayant marqué la culture occidentale comme on pourrait le dire de l'Encyclopédie Diderot et D'Alembert par exemple. Ils n'ont pas été considérés comme fondamentaux dans l'évolution des sciences au XVIII ${ }^{\mathrm{e}}$ ou au XIX ${ }^{\mathrm{e}}$ siècle en Occident. Ils n'étaient pas non plus conçus comme des méthodes destinées à l'apprentissage d'un métier. Malgré cela, les Japonais ont réussi la prouesse de les utiliser comme tels et ont su en tirer de quoi mettre en œuvre et adapter des techniques nouvelles sans l'assistance d'artisans ou d'ingénieurs occidentaux. 

scientifique et technique occidentale dans le milieu des savants puis des artisans au Japon. Cette filtration a permis l'adaptation de nouvelles techniques sur le substrat des savoir-faire artisanaux. L'étude de la réception du travail de Baba Sadayoshi montre aussi qu'il a fallut une période de temps assez longue pour que ces techniques commencent à être mises en pratique, mais que leur incorporation a rendu possible l'apparition d'artisans hautement qualifiés.

Le réexamen des sources et cette comparaison entre le contenu des ouvrages et le développement de nouveaux savoir-faire, ainsi que la comparaison des traductions aux originaux nous amènent à une nouvelle étape de la recherche sur le sujet spécifique de la verrerie à la fin de la période prémoderne. Elle nous permet de re-situer à une juste place l'importance des traductions techniques du tout début du XIX siècle dans l'émergence de la verrerie de type giyaman et l'élaboration de verre d'une composition plus résistante. Elle ramène aussi au centre de ce processus d'adaptation l'importance des associations entre artisans et savants dans la "remise en pratique" des techniques occidentales. Les savants seuls auraient été en peine de passer à l'expérimentation des méthodes décrites sans les connaissances pratiques des artisans et inversement, les verriers ont bénéficié des connaissances intellectuelles des savants. On constate au travers des différents exemples recensés que la demande d'échange émanait tant des artisans que des savants, chacun étant motivés par des nécessités différentes mais convergentes. Baba Sadayoshi a traduit de nombreux livres touchants à des thèmes scientifiques ou techniques très variés. La diversité des traductions réalisées par cette génération de savants des études hollandaises permet de penser qu'une recherche plus poussée sur l'influence de ces traductions scientifiques apporterait un vaste champ d'étude pour une Histoire des techniques au Japon. Une telle étude nous amènerait à mieux connaitre les connexions entre Japon et Occident et leur impact sur les évolutions techniques observées à la fin de la période prémoderne.

\section{NOTES}

1. TOBY Ronald P., « Reopening the Question of Sakoku: Diplomacy in the Legitimation of the Tokugawa Bakufu », Journal of Japanese Studies, vol. 3, n 2, 1977, p. 323-363.

2. KAZUI Tashiro et VIDEEN Susan Downing, « Foreign Relations during the Edo Period: Sakoku Reexamined ", Journal of Japanese Studies, vol. 8, n² 2, 1982, p. 283-306.

3. BABA Sadayoshi, Recueil de méthodes de fabrication du verre (Biidoro seihō shūsetsu), Edo (Tokyo), 1810, manuscrit.

4. BLAIR Dorothy et Corning Museum of Glass, A history of glass in Japan, [1st ed., New York, Kodansha International/USA, 1973, $479 \mathrm{p}$.

5. TANAHASHI Junji, Development of methods of glass-making in Japan from the midth $17^{\text {th }}$ century to the mid-1 th $^{\text {th }}$ century (Kinsei nihon ni okeru garasu seizōhō no hatten to sono genkai), Shoin Kenkyu, nº 1-10, Kobe, 1966-1971.

e-Phaïstos, VI-1 2017| 2018 
6. BLAIR Dorothy et Corning Museum of Glass, A history of glass in Japan, op. cit., p. 236. 7. HENRY William, Udagawa Yōan (trad.), Premiers principes de Chimie (Seimi Kaisō), Edo (Tokyo), 1837.

8. INOUE Akiko, « The Early Development of the Glass Industry of Japan », in Papers on the history of industry and technology of Japan 3, The Development of japanses glass industry, Marburg, Förderverein Marburger Japan-Reihe, 1995, vol. 3/3 p. 12.

9. CHAIKLIN Martha, Cultural commerce and Dutch commercial culture: the influence of European material culture on Japan, 1700-1850, Leiden, Research School CNWS, Leiden University, coll. «Studies in overseas history ", n 5, 2003, p. 116.

10. TANAHASHI Junji, Development of methods of glass-making in Japan from the midth $17^{\text {th }}$ century to the mid-19 ${ }^{\text {th }}$ century (Kinsei nihon ni Okeru garasu seizōhō no hatten to sono genkai), op. cit.

11. BABA Sadayoshi, Recueil de méthodes de fabrication du verre (Biidoro seihō shūsetsu), op. cit.

12. CHALMOT J. A. de, Vervolg op M. Noël Chomel. Algemeen huishoudelyk-, natuur-, zedekundig- en konstwoordenboek [...]. Zynde het VIII.(-XVI.) deel van het woordenboek, Chalmot, Jacques Alexandre de Kampen, 1778-1797 ;Yntema, Jacob Amsterdam, 1762-1799, 1786, $773 \mathrm{p}$.

13. BUYS Egbert, Nieuw en volkomen woordenboek van konsten en weetenschappen: bevattende alle de takken der nuttige kennis ... Alles verzameld uit de beste schryvers in alle taalen, en met een ménigte van nieuwe artykelen vermeerdert, Amsteldam, S.J. Baalde, 1769.

14. PLUCHE Antoine, CLERCQ Pieter Le, GROOT Jan de (Amsterdam), BLUSSE Abraham (Dordrecht) et BLUSSE Pieter (Dordrecht), Schouwtoneel der natuur, of Samenspraaken over de bysonderheden der natuurlyke historie die men geschikt geoordeeld heeft om den jongen lieden leerzucht in te boezemen ..., Te Amsterdam, by J. de Groot, 1776.

15. Ibid., p. 112 et PLUCHE Noël Antoine, Le spectacle de la nature: ou Entretiens sur les particularités de l'histoire naturelle, qui ont paru les plus propres à rendre les jeunes-gens curieux, \& à leur former l'esprit ..., A Paris, Chez la veuve Estienne ... \& Jean Desaint ..., 1732, p. 543. 16. AZUMA Tōru, Sakuma Shōzan et les techniques de la chimie (Sakuma Shōzan to kagaku gijutsu), Kyōto, Shibunkaku Shuppan, 2002, p. 86-90.

17. Traduction de HENRY William, Elements of experimental Chemistry, 1799. UDAGAWA Yōan, Premiers principes de Chimie (Seimi Kaisō), Tokyo, Kodansha, 1837-1847.

18. Lettre à Takata Ikuta, 4 mai 1845, SHŌZAN Sakuma, Euvre complètes de Sakuma Shōzan (Shōzan zenshū), op. cit., p. 313-316.

\section{RÉSUMÉS}

Comment expliquer, dans le contexte de fermeture du Japon prémoderne, en l'absence d'une transmission directe des savoir-faire, les similitudes dans les techniques à l'œuvre et les résultats formels, entre les productions verrières japonaises et occidentales ? Cet article revient sur le rôle des traducteurs dans l'essor de la verrerie au tout début du XIX ${ }^{\mathrm{e}}$ siècle avec une étude du « Recueil de méthodes de fabrication du verre" de Baba Sadayoshi, compilé en 1810. Nous chercherons à savoir quels livres ont été traduits, s'il y a une corrélation entre ces traductions et les techniques 
émergeant à cette période et enfin, quelle a été la diffusion de cet ouvrage et son impact sur les savoir-faire des artisans verriers. Nous verrons ainsi l'étonnant détournement de livres informatifs en " méthodes d'apprentissage » servant à l'émergence d'une nouvelle verrerie ainsi que le rôle des associations entre artisans et savants pour la "remise en pratique» des techniques occidentales.

In the rather secluded environment of early modern Japan, it is unlikely that direct exchanges in the transmission of technique could have occurred between Japanese and Western glass craftsmen. How can it be explained, then, the similarities and parallels in working techniques and formal results between Japanese and Western glass production at the beginning of the 19th century? In order to answer this question, I have chosen to highlight the role of translators in the development of glasswork. I propose this by a study of Baba Sadayoshi's "Compendium of Glassmaking Methods" originally compiled in 1810. This paper presents the Dutch texts chosen by Baba Sadayoshi to compose this compendium. I then check if there is any correlation between these translations and the new techniques used in nineteenth century glass production. Finally, I attempt to measure the diffusion of the "Compendium of Glassmaking Methods" and the impact it may have had on artisan craft skills.

\section{INDEX}

Mots-clés : histoire des techniques, Japon prémoderne, verrerie, circulation des savoirs, rangaku, Baba Sadayoshi

Thèmes : Horizons internationaux

Keywords : history of technology, Early modern Japan, glassmaking, circulation of knowledge, rangaku, Baba Sadayoshi

\section{AUTEUR}

\section{CÉLINE ZURETTI}

Après des études à l'ENSAAMA, Céline Zuretti est devenue artisan-verrier, métier qu'elle a pratiqué quinze ans, en Angleterre, en France et en Espagne, tout en poursuivant des études en Arts plastiques. De retour en France en 2010 afin de poursuivre cette formation à la Sorbonne, elle s'est engagée en parallèle dans les Études Japonaises à l'INALCO. Elle a fait converger ses centres d'intérêts avec une recherche en Histoire des techniques sur « L'Emergence de la verrerie à la période d'Édo au Japon, les dynamiques des transferts de techniques et de connaissances, entre occidentaux, rangaku-sha et artisans ». Son mémoire a obtenu le prix Chrisitan Polak du meilleur mémoire sur le Japon en 2016. Elle effectue depuis une recherche de thèse à Paris Diderot au sein du CRCAO sur « Le Shûseikan du fief de Satsuma - un exemple de première industrialisation à la veille de la Révolution de Meiji » où elle explore des notions telles que la circulation des savoirs techniques ainsi que les dimensions politiques et économiques de ce projet d'industrialisation sur la base des techniques occidentales au Japon. 